\title{
Millimeter-Wave Monitoring of Active Galactic Nuclei with the Africa Millimetre Telescope
}

\author{
Michael Backes ${ }^{1,2, *} \mathbb{D}$, Markus Böttcher ${ }^{2}\left(\mathbb{D}\right.$ and Heino Falcke ${ }^{3,4,5,6}$ \\ 1 Department of Physics, University of Namibia, Private Bag 13301, Windhoek 10005, Namibia \\ 2 Centre for Space Research, North-West University, Private Bag X6001, Potchefstroom 2520, South Africa; \\ Markus.Bottcher@nwu.ac.za \\ 3 Department of Astrophysics/Institute for Mathematics, Astrophysics and Particle Physics (IMAPP), \\ Radboud University, P.O. Box 9010, 6525 AJ Nijmegen, The Netherlands; h.falcke@astro.ru.nl \\ 4 Max-Planck-Institut für Radioastronomie, Auf dem Hügel 69, 53121 Bonn, Germany \\ 5 ASTRON—Netherlands Institute for Radio Astronomy, 7990 AA Dwingeloo, The Netherlands \\ 6 NIKHEF-National Institute for Nuclear and High Energy Physics, 1098 XG Amsterdam, The Netherlands \\ * Correspondence: mbackes@unam.na
}

Received: 27 February 2019; Accepted: 15 June 2019; Published: 25 June 2019

check for updates

\begin{abstract}
Active galactic nuclei are the dominant sources of gamma rays outside our galaxy and are also candidates for the source of ultra-high energy cosmic rays. In addition to being emitters of broad-band non-thermal radiation throughout the electromagnetic spectrum, their emission is highly variable on timescales from years to minutes. Hence, high-cadence monitoring observations are needed to understand their emission mechanisms. The Africa Millimetre Telescope is planned to be the first mm-wave radio telescope on the African continent and one of few in the southern hemisphere. Further to contributing to the global mm-VLBI observations with the Event Horizon Telescope, substantial amounts of observation time will be available for monitoring observations of active galactic nuclei. Here we review the scientific scope of the Africa Millimetre Telescope for monitoring of active galactic nuclei at mm-wavelengths.
\end{abstract}

Keywords: active galactic nuclei; millimeter-wave astronomy; mm-VLBI; monitoring

PACS: 95.85.Bh; 98.54.Cm; 98.54.Gr; 98.62.Nx

\section{Introduction}

Active galactic nuclei (AGN) and more particularly the subclass of blazars have been a major topic of research throughout the electromagnetic spectrum for the past fifty years. Despite significant progress in understanding the blazar phenomenon [1], many open questions remain. Several apparent differences could be attributed to their non-spherical structure and the orientation of the highly relativistic jets relative to the line of sight [2]. Still, the underlying particle acceleration and emission processes remain a matter of current research. Connected to the more than one hundred years of quest for the sources of the highest-energetic cosmic rays, hadronic emission scenarios are tested, e.g., [3,4], and recent coincident gamma-ray and neutrino detections point in that direction [5]. Even though, hadronic emission scenarios are not uncontested, as also the leptonic synchrotron self-Compton (SSC) model [6] is highly successful in explaining the broad-band spectral energy distribution (SED) for blazars, see e.g., [7]. AGN are variable on all time scales: from optical quasi-periods of several years [8] down to gamma-ray flares lasting only minutes [9] and even limiting the size of the emission region to be smaller than $20 \%$ of the gravitational radius of the central black hole [10]. This motivates intensive efforts for monitoring the variability of AGN across the electromagnetic spectrum. 
Following recent positive developments of astronomy in Africa in general [11] and Namibia in particular [12], the Africa Millimetre Telescope (AMT) project aims to build a mm-wave radio telescope in Namibia [13]. It will be the first telescope of its kind on the African continent and one of only few in the Southern hemisphere. The proposed site for the AMT is Mt. Gamsberg $\left(23.34^{\circ} \mathrm{S}, 16.23^{\circ} \mathrm{E}\right)$ in the Khomas Highlands of Namibia, at a height of $2347 \mathrm{~m}$ a.s.l. At very similar latitude to ALMA, this site offers exceptional observing capabilities at $3 \mathrm{~mm}(100 \mathrm{GHz})$ throughout the year as well as strong though seasonal capabilities at $1.3 \mathrm{~mm}(230 \mathrm{GHz})$ and $0.8 \mathrm{~mm}(345 \mathrm{GHz})$, for the latter particularly suited during June through August [13]. The AMT will re-purpose the refurbished structure of the SEST telescope [14] and shall (initially) employ receivers for $3.5 \mathrm{~mm}$ and $1.3 \mathrm{~mm}(86 \mathrm{GHz}$ and $230 \mathrm{GHz})$, possibly extended to $0.8 \mathrm{~mm}(345 \mathrm{GHz})$. One of the main scientific drivers for the AMT are observations of the 'shadows' of the black holes at the centres of our Milky Way, Sagittarius A*, and of the radio galaxy M 87 at $3.5 \mathrm{~mm}$ and $1.3 \mathrm{~mm}$ within the Event Horizon Telescope (EHT) network [15]. The shadow of the black hole at the centre of M 87 has recently been imaged for the first time by the EHT [16]. Initial simulations for Sagittarius $A^{*}$ indicate a significant improvement in image quality and, hence, angular resolution by adding the AMT to the EHT [17]. Still, EHT observations will only make use of a small fraction of the available observation time on the AMT, allowing for high-cadence monitoring observations of AGN to be conducted in addition.

\section{Scientific Rationale}

Particularly because of their high variability, monitoring of AGN is crucial to obtain a complete picture of their variability patterns and to understand the underlying phenomena in these enigmatic objects. Across all accessible wavelength regimes, monitoring campaigns are being conducted; besides the all-sky monitoring in X-rays by MAXI, aboard the International Space Station, and Swift-BAT, in high energy gamma rays by AGILE-GRID and Fermi-LAT and in ultra-high energy gamma rays by HAWC, targeted monitoring of AGN is conducted particularly successfully by optical telescopes, e.g., SMARTS [18], GASP [19] of the WEBT [20], and the Steward Observatory blazar monitoring program [21], radio telescopes, e.g., OVRO [22], UMRAO [23], and Metsähovi [24,25], and the imaging atmospheric Cherenkov telescopes (IACTs) in the very-high energy gamma rays. VERITAS and MAGIC pursue long-term blazar monitoring [26,27] and FACT $[28,29]$ was built for the purpose of blazar monitoring [30-32], even with the idea in mind to set up a world-wide network for continuous monitoring [33]. In the southern hemisphere, the H.E.S.S. telescopes conduct very-high energy gamma-ray monitoring of AGN [34,35]. These IACT observations are regularly complemented by optical monitoring in $R_{C^{-}}$and $B_{C}$-bands by the robotic KVA [36] and ATOM telescopes [37,38].

It has long been established by very long baseline interferometry (VLBI) at up to $43 \mathrm{GHz}$ (7 mm) that the high energy gamma-ray emission as measured by CGRO-EGRET is coincident with the appearance of new VLBI features ('knots') [39]. Later, this was underpinned with the observation of a gamma-ray flare coincident with a new VLBI knot in the radio galaxy M 87 [40]. In this context, there have been long-standing programs VLBI flux and morphology monitoring of AGN like MOJAVE in the northern hemisphere at $15 \mathrm{GHz}(2 \mathrm{~cm})$ [41], TANAMI in the southern hemisphere at $22 \mathrm{GHz}$ $(1.3 \mathrm{~cm})$ [42], and VLBA-BU-BLAZAR in the northern hemisphere at $43 \mathrm{GHz}(7 \mathrm{~mm})$ [43]. Further, single-dish flux monitoring has been conducted at up to $43 \mathrm{GHz}$ with the $100 \mathrm{~m}$ Effelsberg telescope within F-GAMMA [44,45] and with APEX at $345 \mathrm{GHz}(0.87 \mathrm{~mm})$ [46]. Polarimetric monitoring at $86 \mathrm{GHz}$ and $229 \mathrm{GHz}(3.5 \mathrm{~mm}$ and $1.3 \mathrm{~mm})$ has been conducted in the northern hemisphere at the IRAM $30 \mathrm{~m}$ telescope within the POLAMI programme [47-49]. Further, multi-wavelengths monitoring campaigns have been organized, like MARMOT [50]. A recent review of mm-VLBI observations of AGN is given in [51], whereas the major initiative of mm-VLBI monitoring of AGN is conducted by the GMVA [52] at $86 \mathrm{GHz}(3.5 \mathrm{~mm})$ and the EHT at higher frequencies. For both initiatives, the only telescopes in the southern hemisphere are (phased) ALMA and APEX (in Chile) and the South Pole Telescope. Whereas the addition of another Southern hemisphere telescope to the EHT is one of the major and obvious scientific drivers of the AMT, complementing the GMVA at $3.5 \mathrm{~mm}$ VLBI 
and improving the $u-v$-coverage of mm-VLBI observations of Southern AGN is a scientific purpose in its own right; with the superior angular resolution of $\mathrm{mm}$-VLBI observations over $\mathrm{cm}$-VLBI the smallest scale structures, closest to the core of AGN can be resolved. Combined with polarization imaging, the ordering and mean direction of the magnetic field at the base of the jet can be determined to distinguish between models of highly ordered helical magnetic fields or turbulent ones (possibly with standing shocks), e.g., [53,54].

Though correlation studies between $5 \mathrm{GHz}-8 \mathrm{GHz}$ VLBI flux densities obtained from the Radio Fundamental Catalogue and Fermi-LAT measurements show much stronger correlation for the high energy $(>100 \mathrm{MeV})$ measurements than for the very-high energy $(>50 \mathrm{GeV})$ ones [55], preliminary findings comparing ALMA observations at $230 \mathrm{GHz}(1.3 \mathrm{~mm})$ of a sample of 77 AGN from the Fermi-LAT 3FGL catalogue (above $100 \mathrm{MeV}$ ) show a significantly higher correlation than comparing the Fermi-LAT flux to $1.4 \mathrm{GHz}$ data [56]. This hints at the mm-wave emission of AGN being more strongly connected to the gamma-ray emission than the cm-wave emission. The AMT will for the first time allow for strictly simultaneous observations of mm-wave and very-high energy gamma-ray emission, due to the close location of the AMT to the H.E.S.S. telescopes, which will allow for unprecedented studies of the emission properties of Southern hemisphere AGN.

One of the major drawbacks of $\mathrm{cm}$ radio flux density observations of AGN is that the $\mathrm{cm}$ emission is likely produced at different regions of the jet and, hence, for consistent modelling of the spectral energy distribution, these measurements have to be ignored because of the opaqueness of the emission region to $\mathrm{cm}$ radio waves due to synchrotron self-absorption. This changes drastically for observations in the mm-regime. Likely for the $3.5 \mathrm{~mm}(86 \mathrm{GHz})$ emission but certainly for the $1.3 \mathrm{~mm}(230 \mathrm{GHz})$ one, synchrotron self-absorption is negligible and the innermost regions of the jet can be probed. Such observations could test whether mm-wave and (very-)high energy emission are produced co-spatially. In this case, both, spectral as well as temporal studies would be significantly enriched. Not only would the mm-wave flux be useful for constraining AGN emission models, but also temporal correlation of the respective lightcurves would be expected-obeying differences in the electron cooling timescales. The electron cooling timescale for synchrotron emission at $230 \mathrm{GHz}$ is given by

$$
t_{c}(\text { obs }) \sim 40 B_{G}^{-3 / 2} \delta_{1}^{-1 / 2} \text { days, }
$$

where $B_{G}$ is the strength of the magnetic field in Gauss and $\delta_{1}$ is the Doppler factor in units of 10 . It is rather well established that emission of AGN close to the core is produced in confined volumes with $R \lesssim 10^{16} \mathrm{~cm}$, which are moving at relativistic speed $\beta_{\Gamma}=\sqrt{1-\frac{1}{\Gamma^{2}}}$, with a bulk Lorentz factor $\Gamma$, along the jet. The Doppler factor is defined as $\delta \equiv \frac{1}{\Gamma\left(1-\beta_{\Gamma} \cos \theta^{\mathrm{obs}}\right)}$, with $\theta^{\text {obs }}$ being the viewing angle (with respect to the jet axis) in the observer's rest frame. Considering that neither the magnetic field strength nor the Doppler factor are known and taking into account the possibility for Compton-dominated cooling, resulting in shorter cooling timescales than given in Equation (1), this well motivates monitoring observations with weekly cadence. For emission of visual light, the cooling timescale is shorter by a factor of $\sim 100$ than given in Equation (1) above. Hence, for co-spatial emission, the optical light curves should lead the radio ones by this difference. Further, if such a time-lag could be established (assuming an independent estimate of the Doppler factor), the magnetic field strength in the emission region could be estimated (as proposed initially for intra-optical time-lags in [57]). The magnetic field is generally believed to play a central role in the launching and collimation of AGN jets and in the acceleration of relativistic electrons. Thus, probing the magnetic field in the mm-emitting region will aid in clarifying its role in the particle acceleration (magnetic reconnection, e.g., [54], vs. shocks, e.g., [58], vs. shear layers, e.g., [59]) and its potential for collimating the jet.

Even if no correlation with identifiable time lag between $\mathrm{mm}$ and optical flux is observed, the mm-wave monitoring observations would be highly useful; in case the mm-wave variability would not be correlated to the optical variability of a given AGN, this would indicate different emission regions. Possibly, molecular and dust emissions could not only dominate the infrared part of the spectral energy 
distribution but even the mm-wave emission, as observed for some blazars [60]. This, in turn, would drastically help to constrain external photon fields within the AGN as invoked for external-Compton emission models [61].

Obviously, for any significant correlation studies of multi-wavelengths lightcurves, high-cadence monitoring observations are needed as will be supplied by the AMT.

\section{Summary}

Summarizing, there is ample scope for the AMT to have significant impact in the field of (gamma-ray loud) AGN. Particularly, high-cadence single dish monitoring observations at $1-3.5 \mathrm{~mm}$ will help to constrain theoretical models of emission mechanisms as well the the site of gamma-ray production. Even more so, $3.5 \mathrm{~mm}$ VLBI observations with the GMVA and $1.3 \mathrm{~mm}$ VLBI observations with the EHT will help resolving the emission features and hence, foster the understanding of the structure and formation processes of AGN jets.

Author Contributions: Conceptualization, M.B. (Michael Backes) and H.F.; writing-original draft preparation, M.B. (Michael Backes); writing—review and editing, M.B. (Michael Backes), M.B. (Markus Böttcher), and H.F.; project administration, H.F.; funding acquisition, H.F. and M.B. (Markus Böttcher).

Funding: This work is partly supported by the ERC Synergy Grant "BlackHoleCam: Imaging the Event Horizon of Black Holes" (Grant 610058) and the National Research Foundation (NRF) ${ }^{1}$ and the Department of Science and Technology of the Republic of South Africa through the South African Research Chair Initiative under SARChI grant 64798 .

Acknowledgments: M.B. (Michael Backes) wants to thank Alan Marscher and Svetlana Jorstad for fruitful discussions.

Conflicts of Interest: The authors declare no conflict of interest.

\section{Abbreviations}

The following abbreviations are used in this manuscript (in order of appearance):

AGN Active galactic nucleus

SSC Synchrotron self-Compton

SED Spectral energy distribution

AMT Africa Millimetre Telescope, https://www.ru.nl/blackhole/africa-millimetre-telescope

ALMA Atacama Large Millimeter/submillimeter Array, https://www.almaobservatory.org

SEST Swedish ESO Submillimetre Telescope, http:/ /www.apex-telescope.org/sest

EHT Event Horizon Telescope, https://eventhorizontelescope.org

MAXI Monitor of All-sky X-ray Image, aboard the International Space Station,

http:/ / maxi.riken.jp/top

Swift-BAT Burst Alert Telescope, aboard the Neil Gehrels Swift Observatory,

https: / / swift.gsfc.nasa.gov

AGILE-GRID Gamma Ray Imaging Detector, aboard the Astro-Rivelatore Gamma a Immagini

Leggero satellite, http:/ / agile.rm.iasf.cnr.it

Fermi-LAT Large Array Telescope, aboard the Fermi Gamma-ray Space Telescope,

https: / / fermi.gsfc.nasa.gov

HAWC High-Altitude Water Cherenkov Observatory, https://www.hawc-observatory.org

SMARTS Small and Moderate Aperture Research Telescope System,

http:/ / www.astro.yale.edu/smarts / glast

GASP GLAST-AGILE Support Programme,

http:/ / www.oato.inaf.it/blazars/webt/gasp/homepage.html

WEBT Whole Earth Blazar Telescope, http://www.oato.inaf.it/blazars/webt

OVRO Owens Valley Radio Observatory, http://www.astro.caltech.edu/ovroblazars

1 Any opinion, finding and conclusion or recommendation expressed in this material is that of the authors and the NRF does not accept any liability in this regard. 


$\begin{array}{ll}\text { UMRAO } & \text { University of Michigan Radio Astronomy Observatory, } \\ \text { IACT } & \text { https://dept.astro.lsa.umich.edu/datasets/umrao.php } \\ \text { VERITAS } & \text { Very Energetic Radiation Imaging Telescope Array System, } \\ & \text { https://veritas.sao.arizona.edu } \\ \text { MAGIC } & \text { Major Atmospheric Gamma Imaging Cherenkov, https://magic.mpp.mpg.de } \\ \text { H.E.S.S. } & \text { High Energy Stereoscopic System, https://www.mpi-hd.mpg.de/hfm/HESS } \\ \text { KVA } & \text { http://users.utu.fi//kani/1m } \\ \text { ATOM } & \text { Automatic Telescope for Optical Monitoring, } \\ & \text { https://www.lsw.uni-heidelberg.de/projects/hess/ATOM } \\ \text { VLBI } & \text { Very long baseline interferometry } \\ \text { CGRO-EGRET } & \text { Energetic Gamma Ray Experiment Telescope, aboard the Compton Gamma Ray } \\ & \text { Observatory, https://heasarc.gsfc.nasa.gov/docs/cgro/cgro/egret.html } \\ \text { MOJAVE } & \text { Monitoring Of Jets in Active galactic nuclei with VLBA Experiments, } \\ \text { TANAMI } & \text { http://www.physics.purdue.edu/MOJAVE } \\ \text { Tracking Active galactic Nuclei with Austral Milliarcsecond Interferometry, } \\ \text { F-GAMMA } & \text { http://pulsar.sternwarte.uni-erlangen.de/tanami } \\ \text { FERMI-GST AGN Multi-frequency Monitoring Alliance, } \\ \text { VLBA } & \text { https://www3.mpifr-bonn.mpg.de/div/vlbi/fgamma/fgamma.html } \\ \text { VLBA-BU-BLAZAR } & \text { Very Long Baseline Array, https://science.lbo.us/facilities/vlba } \\ \text { VLBA Boston University Blazar monitoring project, } \\ \text { APEX } & \text { http://www.bu.edu/blazars/VLBAproject.html } \\ \text { PRAM } & \text { Atacama Pathfinder EXperiment, http://www.apex-telescope.org } \\ \text { MARMOT } & \text { Institut de Radioastronomie Millimétrique, http://iram-institute.org } \\ \text { GMVA } & \text { POLarimetric monitoring of AGN at MIllimetre Wavelengths, http://polami.iaa.es } \\ & \text { Monitoring of } \gamma \text {-ray Active galactic nuclei with Radio, Millimetre and Optical } \\ & \text { Telescopes, http://www.astro.caltech.edu/marmot } \\ \text { Global mm-VLBI Array, https://www3.mpifr-bonn.mpg.de/div/vlbi/globalmm }\end{array}$

\section{References}

1. Böttcher, M. Progress in Multi-wavelength and Multi-Messenger Observations of Blazars and Theoretical Challenges. Galaxies 2019, 7, 20. [CrossRef]

2. Padovani, P.; Urry, C.M. Luminosity functions, relativistic beaming, and unified theories of high-luminosity radio sources. Astrophys. J. 1992, 387, 449-457. [CrossRef]

3. Böttcher, M. A Hadronic Synchrotron Mirror Model for the "Orphan" TeV Flare in 1ES 1959+650. Astrophys. J. 2005, 621, 176-180. [CrossRef]

4. Reimer, A.; Böttcher, M.; Postnikov, S. Neutrino Emission in the Hadronic Synchrotron Mirror Model: The “Orphan" TeV Flare from 1ES 1959+650. Astrophys. J. 2005, 630, 186-190. [CrossRef]

5. IceCube Collaboration; Aartsen, M.G.; Ackermann, M.; Fermi-LAT; MAGIC; AGILE; ASAS-SN; HAWC; H.E.S.S.; INTEGRAL; et al. Multimessenger observations of a flaring blazar coincident with high-energy neutrino IceCube-170922A. Science 2018, 361, eaat1378. [CrossRef]

6. Tavecchio, F.; Maraschi, L.; Ghisellini, G. Constraints on the Physical Parameters of TeV Blazars. Astrophys. J. 1998, 509, 608-619. [CrossRef]

7. Abdo, A.A.; Ackermann, M.; Ajello, M.; Baldini, L.; Ballet, J.; Barbiellini, G.; Bastieri, D.; Bechtol, K.; Bellazzini, R.; Berenji, B.; et al. Fermi Large Area Telescope Observations of Markarian 421: The Missing Piece of its Spectral Energy Distribution. Astrophys. J. 2011, 736, 131. [CrossRef]

8. Sillanpaa, A.; Haarala, S.; Valtonen, M.J.; Sundelius, B.; Byrd, G.G. OJ 287-Binary pair of supermassive black holes. Astrophys. J. 1988, 325, 628-634. [CrossRef]

9. Aharonian, F.; Akhperjanian, A.G.; Bazer-Bachi, A.R.; Behera, B.; Beilicke, M.; Benbow, W.; Berge, D.; Bernlöhr, K.; Boisson, C.; Bolz, O.; et al. An Exceptional Very High Energy Gamma-Ray Flare of PKS 2155-304. Astrophys. J. Lett. 2007, 664, L71-L74. [CrossRef] 
10. Aleksić, J.; Ansoldi, S.; Antonelli, L.A.; Antoranz, P.; Babic, A.; Bangale, P.; Barrio, J.A.; González, J.B.; Bednarek, W.; Bernardini, E.; et al. Black hole lightning due to particle acceleration at subhorizon scales. Science 2014, 346, 1080-1084. [CrossRef]

11. Pović, M.; Backes, M.; Baki, P.; Baratoux, D.; Tessema, S.B.; Benkhaldoun, Z.; Bode, M.; Klutse, N.A.B.; Charles, P.; Govender, K.; et al. Development in astronomy and space science in Africa. Nat. Astron. 2018, 2,507-510. [CrossRef]

12. Backes, M.; Evans, R.; Kasai, E.K.; Steenkamp, R. Status of Astronomy in Namibia. Afr. Rev. Phys. 2018, 13, 90-95.

13. Backes, M.; Müller, C.; Conway, J.E.; Deane, R.; Evans, R.; Falcke, H.; Fraga-Encinas, R.; Goddi, C.; Klein Wolt, M.; Krichbaum, T.P.; et al. The Africa Millimetre Telescope. In Proceedings of the 4th Annual Conference on High Energy Astrophysics in Southern Africa (HEASA 2016), Cape Town, South Africa, 25-26 August 2016; South African Astronomical Observatory (SAAO): Cape Town, South Africa, 2016; p. 29. [CrossRef]

14. Booth, R.S.; Delgado, G.; Hagstrom, M.; Johansson, L.E.B.; Murphy, D.C.; Olberg, M.; Whyborn, N.D.; Greve, A.; Hansson, B.; Lindstrom, C.O.; et al. The Swedish-ESO Submillimetre Telescope (SEST). Astron. Astrophys. 1989, 216, 315-324.

15. Event Horizon Telescope Collaboration; Akiyama, K.; Alberdi, A.; Alef, W.; Asada, K.; Azulay, R.; Baczko, A.-K.; Ball, D.; Baloković, M.; Barrett, J.; et al. First M87 Event Horizon Telescope Results. II. Array and Instrumentation. Astrophys. J. Lett. 2019, 875, L2. [CrossRef]

16. Event Horizon Telescope Collaboration; Akiyama, K.; Alberdi, A.; Gómez Fernández, J.L. First M87 Event Horizon Telescope Results. I. The Shadow of the Supermassive Black Hole. Astrophys. J. Lett. 2019, 875, L1. [CrossRef]

17. Mizuno, Y.; Younsi, Z.; Fromm, C.M.; Porth, O.; De Laurentis, M.; Olivares, H.; Falcke, H.; Kramer, M.; Rezzolla, L. The current ability to test theories of gravity with black hole shadows. Nat. Astron. 2018, 2, 585-590. [CrossRef]

18. Bonning, E.; Urry, C.M.; Bailyn, C.; Buxton, M.; Chatterjee, R.; Coppi, P.; Fossati, G.; Isler, J.; Maraschi, L. SMARTS Optical and Infrared Monitoring of 12 Gamma-Ray Bright Blazars. Astrophys. J. 2012, 756, 13. [CrossRef]

19. Villata, M. The GLAST-AGILE Support Program of the Whole Earth Blazar Telescope. Accretion and Ejection in AGN: A Global View. In Astronomical Society of the Pacific Conference Series; Maraschi, L., Ghisellini, G., Della Ceca, R., Tavecchio, F., Eds.; Astronomical Society of the Pacific: San Francisco, CA, USA, 2010; Volume 427, p. 308.

20. Villata, M.; Raiteri, C.M.; Tosti, G.; Ciprini, S.; Ibrahimov, M.A.; Kurtanidze, O.M.; Massaro, E.; Mattox, J.R.; Nesci, R.; Nikolashvili, M.G.; et al. The Whole Earth Blazar Telescope (WEBT). Mem. Della Societa Astron. Ital. 2002, 73, 1191-1192.

21. Smith, P.S.; Montiel, E.; Rightley, S.; Turner, J.; Schmidt, G.D.; Jannuzi, B.T. Coordinated Fermi/Optical Monitoring of Blazars and the Great 2009 September Gamma-ray Flare of 3C 454.3. In Proceedings of the 2009 Fermi Symposium, eConf Proceedings C09112, Washington, DC, USA, 2-5 November 2009.

22. Richards, J.L.; Max-Moerbeck, W.; Pavlidou, V.; King, O.G.; Pearson, T.J.; Readhead, A.C.S.; Reeves, R.; Shepherd, M.C.; Stevenson, M.A.; Weintraub, L.C.; et al. Blazars in the Fermi Era: The OVRO $40 \mathrm{~m}$ Telescope Monitoring Program. Astrophys. J. Suppl. 2011, 194, 29. [CrossRef]

23. Aller, M.; Aller, H.; Hughes, P. The University of Michigan Centimeter-Band All Stokes Blazar Monitoring Program: Single-Dish Polarimetry as a Probe of Parsec-Scale Magnetic Fields. Galaxies 2017, 5, 75. [CrossRef]

24. Terasranta, H.; Tornikoski, M.; Valtaoja, E.; Urpo, S.; Nesterov, N.; Lainela, M.; Kotilainen, J.; Wiren, S.; Laine, S.; Nilsson, K.; et al. Ten years monitoring of extragalactic radio sources at 22, 37 and $87 \mathrm{GHz}$. Astron. Astrophys. Suppl. 1992, 94, 121-173.

25. Teräsranta, H.; Wiren, S.; Koivisto, P.; Saarinen, V.; Hovatta, T. 24 year monitoring of extragalactic sources at 22 and $37 \mathrm{GHz}$. Astron. Astrophys. 2005, 440, 409-410. [CrossRef]

26. Goebel, F.; Backes, M.; Bretz, T.; Hayashida, M.; Hsu, C.; Mannheim, K.; Moralejo, A.; Rhode, W.; Satalecka, K.; Shayduk, M.; et al. Long term monitoring of bright TeV Blazars with the MAGIC telescope. In Proceedings of the 30th International Cosmic Ray Conference ICRC, Mérida, Mexico, 3-11 July 2007; Volume 3, pp. 1025-1028. 
27. Wagner, R.; Backes, M.; Satalecka, K.; Bonnoli, G.; Doert, M.; Steinke, B.; Strah, N.; Terzic, T.; Tescaro, D.; Uellenbeck, M.; et al. Monitoring of bright, nearby Active Galactic Nuclei with the MAGIC telescopes. In Proceedings of the 32nd International Cosmic Ray Conference, Beijing, China, 11-18 August 2011; Volume 8, pp. 145-148. [CrossRef]

28. Anderhub, H.; Backes, M.; Biland, A.; Boller, A.; Braun, I.; Bretz, T.; Commichau, S.; Commichau, V.; Domke, M.; Dorner, D.; et al. FACT-The first Cherenkov telescope using a G-APD camera for TeV gamma-ray astronomy. Nucl. Instrum. Methods Phys. Res. A 2011, 639, 58-61. [CrossRef]

29. Anderhub, H.; Backes, M.; Biland, A.; Boccone, V.; Braun, I.; Bretz, T.; Buß, J.; Cadoux, F.; Commichau, V.; Djambazov, L.; et al. Design and operation of FACT-The first G-APD Cherenkov telescope. J. Instrum. 2013, 8, P06008. [CrossRef]

30. Bretz, T.; Backes, M.; Rhode, W.; Mannheim, K.; Becker, J.; Dorner, D.; Kneiske, T.; Meyer, M. Long-term VHE $\gamma$-ray monitoring of bright blazars with a dedicated Cherenkov telescope. In Proceedings of the 30th International Cosmic Ray Conference, Mérida, Mexico, 3-11 July 2007; Voume 3, pp. 1495-1498.

31. Backes, M.; Becker, J.K.; Clevermann, F.; Neise, D.; Rhode, W.; Rollke, S.; Pohl, M.; Ribordy, M.; Renker, D.; Bretz, T.; et al. Long-Term Monitoring of Bright Blazars with a Dedicated Cherenkov Telescope. Int. J. Mod. Phys. D 2009, 18, 1645-1649. [CrossRef]

32. Temme, F.; Adam, J.; Ahnen, M.; Baack, D.; Balbo, M.; Bergmann, M.; Biland, A.; Blank, M.; Bretz, T.; Brügge, K.; et al. Long-Term Monitoring of Bright Blazars in the Multi-GeV to TeV Range with FACT. Galaxies 2017, 5, 18. [CrossRef]

33. Backes, M.; Biland, A.; Boller, A.; Braun, I.; Bretz, T.; Commichau, S.; Commichau, V.; Dorner, D.; von Gunten, H.; Gendotti, A.; et al. Long-term monitoring of blazars-The DWARF network. In Proceedings of the 31st International Cosmic Ray Conference, Łódź, Poland, 7-15 July 2009; p. 1452.

34. H. E. S. S. Collaboration; Abramowski, A.; Aharonian, F.; Ait Benkhali, F.; Akhperjanian, A.G.; Angüner, E.O.; Backes, M.; Balenderan, S.; Balzer, A.; Barnacka, A.; et al. Long-term monitoring of PKS 2155-304 with ATOM and H.E.S.S.: Investigation of optical $/ \gamma$-ray correlations in different spectral states. Astron. Astrophys. 2014, 571, A39. [CrossRef]

35. Zacharias, M.; Jankowsky, F.; Mohamed, M.; Prokoph, H.; Sanchez, D.; Wagner, S.; Wierzcholska, A. Monitoring of the FSRQ PKS 1510-089 with H.E.S.S. In Proceedings of the 35th International Cosmic Ray Conference (ICRC 2017), Busan, Korea, 10-20 July 2017; Volume ICRC2017, p. 654. [CrossRef]

36. Nilsson, K.; Lindfors, E.; Takalo, L.O.; Reinthal, R.; Berdyugin, A.; Sillanpää, A.; Ciprini, S.; Halkola, A.; Heinämäki, P.; Hovatta, T.; et al. Long-term optical monitoring of TeV emitting blazars. I. Data analysis. Astron. Astrophys. 2018, 620, A185. [CrossRef]

37. Hauser, M.; Möllenhoff, C.; Pühlhofer, G.; Wagner, S.J.; Hagen, H.J.; Knoll, M. ATOM-An Automatic Telescope for Optical Monitoring. Astron. Nachr. 2004, 325, 659. [CrossRef]

38. Wierzcholska, A.; Ostrowski, M.; Stawarz, Ł.; Wagner, S.; Hauser, M. Longterm optical monitoring of bright BL Lacertae objects with ATOM: Spectral variability and multiwavelength correlations. Astron. Astrophys. 2015, 573, A69. [CrossRef]

39. Jorstad, S.G.; Marscher, A.P.; Mattox, J.R.; Wehrle, A.E.; Bloom, S.D.; Yurchenko, A.V. Multiepoch Very Long Baseline Array Observations of EGRET-detected Quasars and BL Lacertae Objects: Superluminal Motion of Gamma-Ray Bright Blazars. Astrophys. J. Suppl. 2001, 134, 181-240. [CrossRef]

40. Acciari, V.A.; Aliu, E.; Arlen, T.; Bautista, M.; Beilicke, M.; Benbow, W.; Bradbury, S.M.; Buckley, J.H.; Bugaev, V.; Bugaev, Y.; et al. Radio Imaging of the Very-High-Energy $\gamma$-Ray Emission Region in the Central Engine of a Radio Galaxy. Science 2009, 325, 444-448. [CrossRef] [PubMed]

41. Lister, M.L.; Homan, D.C. MOJAVE: Monitoring of Jets in Active Galactic Nuclei with VLBA Experiments. I. First-Epoch 15 GHz Linear Polarization Images. Astron. J. 2005, 130, 1389-1417. [CrossRef]

42. Ojha, R.; Kadler, M.; Böck, M.; Booth, R.; Dutka, M.S.; Edwards, P.G.; Fey, A.L.; Fuhrmann, L.; Gaume, R.A.; Hase, H.; et al. TANAMI: Tracking active galactic nuclei with austral milliarcsecond interferometry. I. First-epoch $8.4 \mathrm{GHz}$ images. Astron. Astrophys. 2010, 519, A45. [CrossRef]

43. Jorstad, S.; Marscher, A. The VLBA-BU-BLAZAR Multi-Wavelength Monitoring Program. Galaxies 2016, 4, 47. [CrossRef] 
44. Fuhrmann, L.; Angelakis, E.; Zensus, J.A.; Nestoras, I.; Marchili, N.; Pavlidou, V.; Karamanavis, V.; Ungerechts, H.; Krichbaum, T.P.; Larsson, S.; et al. The F-GAMMA programme: Multi-frequency study of active galactic nuclei in the Fermi era. Programme description and the first 2.5 years of monitoring. Astron. Astrophys. 2016, 596, A45. [CrossRef]

45. Angelakis, E.; Fuhrmann, L.; Myserlis, I.; Zensus, J.A.; Nestoras, I.; Karamanavis, V.; Marchili, N.; Krichbaum, T.P.; Kraus, A.; Rachen, J.P. F-GAMMA: Multi-frequency radio monitoring of Fermi blazars. The 2.64 to 43 GHz Effelsberg light curves from 2007-2015. Astron. Astrophys. 2019, 626, A60. [CrossRef]

46. Larsson, S.; Fuhrmann, L.; Weiss, A.; Angelakis, E.; Krichbaum, T.P.; Nestoras, I.; Zensus, J.A.; Axelsson, M.; Nilsson, D.; Ryde, F.; et al. APEX sub-mm monitoring of gamma-ray blazars. In Proceedings of the Conference Fermi and Jansky: Our Evolving Understanding of AGN-eConf C1111101, St Michaels, MD, USA, 10-12 November 2011; Harbourtowne Conference Center: St Michaels, MD, USA, 2012.

47. Agudo, I.; Thum, C.; Molina, S.N.; Casadio, C.; Wiesemeyer, H.; Morris, D.; Paubert, G.; Gómez, J.L.; Kramer, C. POLAMI: Polarimetric Monitoring of AGN at Millimetre Wavelengths - I. The programme, calibration and calibrator data products. Mon. Not. R. Astron. Soc. 2018, 474, 1427-1435. [CrossRef]

48. Thum, C.; Agudo, I.; Molina, S.N.; Casadio, C.; Gómez, J.L.; Morris, D.; Ramakrishnan, V.; Sievers, A. POLAMI: Polarimetric Monitoring of Active Galactic Nuclei at Millimetre Wavelengths-II. Widespread circular polarization. Mon. Not. R. Astron. Soc. 2018, 473, 2506-2520. [CrossRef]

49. Agudo, I.; Thum, C.; Ramakrishnan, V.; Molina, S.N.; Casadio, C.; Gómez, J.L. POLAMI: Polarimetric Monitoring of Active Galactic Nuclei at Millimetre Wavelengths-III. Characterization of total flux density and polarization variability of relativistic jets. Mon. Not. R. Astron. Soc. 2018, 473, 1850-1867. [CrossRef]

50. Ramakrishnan, V.; Hovatta, T.; Tornikoski, M.; Nilsson, K.; Lindfors, E.; Baloković, M.; Lähteenmäki, A.; Reinthal, R.; Takalo, L. Locating the $\gamma$-ray emission site in Fermi/LAT blazars-II. Multifrequency correlations. Mon. Not. R. Astron. Soc. 2016, 456, 171-180. [CrossRef]

51. Boccardi, B.; Krichbaum, T.P.; Ros, E.; Zensus, J.A. Radio observations of active galactic nuclei with mm-VLBI. Astron. Astrophys. Rev. 2017, 25, 4. [CrossRef]

52. Nair, D.G.; Lobanov, A.P.; Krichbaum, T.P.; Ros, E.; Zensus, J.A.; Kovalev, Y.Y.; Lee, S.S.; Mertens, F.; Hagiwara, Y.; Bremer, M.; et al. Global millimeter VLBI array survey of ultracompact extragalactic radio sources at $86 \mathrm{GHz}$. Astron. Astrophys. 2019, 622, A92. [CrossRef]

53. Marscher, A.P. Turbulent, Extreme Multi-zone Model for Simulating Flux and Polarization Variability in Blazars. Astrophys. J. 2014, 780, 87. [CrossRef]

54. Sironi, L.; Petropoulou, M.; Giannios, D. Relativistic jets shine through shocks or magnetic reconnection? Mon. Not. R. Astron. Soc. 2015, 450, 183-191. [CrossRef]

55. Lico, R.; Giroletti, M.; Orienti, M.; Costamante, L.; Pavlidou, V.; D’Ammando, F.; Tavecchio, F. Exploring the connection between radio and $\mathrm{GeV}-\mathrm{TeV} \gamma$-ray emission in the 1FHL and $2 \mathrm{FHL}$ AGN samples. Astron. Astrophys. 2017, 606, A138. [CrossRef]

56. Giroletti, M.; D'Ammando, F.; Orienti, M.; Lico, R. The Radio/Gamma-Ray Connection from $120 \mathrm{MHz}$ to $230 \mathrm{GHz}$. Galaxies 2016, 4, 30. [CrossRef]

57. Böttcher, M.; Marscher, A.P.; Ravasio, M.; Villata, M.; Raiteri, C.M.; Aller, H.D.; Aller, M.F.; Teräsranta, H.; Mang, O.; Tagliaferri, G.; et al. Coordinated Multiwavelength Observations of BL Lacertae in 2000. Astrophys. J. 2003, 596, 847-859. [CrossRef]

58. Marscher, A.P.; Gear, W.K. Models for high-frequency radio outbursts in extragalactic sources, with application to the early 1983 millimeter-to-infrared flare of 3C 273. Astrophys. J. 1985, 298, 114-127. [CrossRef]

59. Liang, E.; Fu, W.; Böttcher, M.; Roustazadeh, P. Scaling of Relativistic Shear Flows with the Bulk Lorentz Factor. Astrophys. J. 2018, 854, 129. [CrossRef]

60. Fumagalli, M.; Dessauges-Zavadsky, M.; Furniss, A.; Prochaska, J.X.; Williams, D.A.; Kaplan, K.; Hogan, M. A search of $\mathrm{CO}$ emission lines in blazars: The low molecular gas content of BL Lac objects compared to quasars. Mon. Not. R. Astron. Soc. 2012, 424, 2276-2283. [CrossRef]

61. Dermer, C.D.; Schlickeiser, R. Model for the High-Energy Emission from Blazars. Astrophys. J. 1993, 416, 458. [CrossRef]

(C) 2019 by the authors. Licensee MDPI, Basel, Switzerland. This article is an open access article distributed under the terms and conditions of the Creative Commons Attribution (CC BY) license (http:/ / creativecommons.org/licenses/by/4.0/). 